\title{
Enhanced Charge Transport in 2D materials through Polaritonic States
}

\author{
Pooja Bhatt, Kuljeet Kaur and Jino George* \\ Indian Institute of Science Education and Research (IISER) Mohali, Punjab-140306, India.
}

\begin{abstract}
Strong light-matter interaction of functional materials has opened up an unexplored area of research to effectively utilize the exotic behavior of polaritonic states. Recent experiments suggest that material properties like charge transport can be controlled in strongly coupled systems. Fabry-Perot cavities based on metal mirrors pose a major challenge in reading out the electrical properties of hybrid states. Here, we explored the design of a one-mirror Fabry-Perot cavity and studied charge transport of 2D materials in a field-effect transistor configuration. The optical and electrical signature of strongly coupled few-layer WS2 suggest an increase in electron transport. Charge transport mobility is enhanced by $\sim 50$ times under ON reso-nance condition. A clear correlation in the effective mass of the polaritonic state and Schottky barrier height may be indicating a coherent nature of light-matter interaction.
\end{abstract}

KEYWORDS: Charge Transport, 2D materials, Polaritonic States, Light-matter Strong Coupling, Polaritronics.
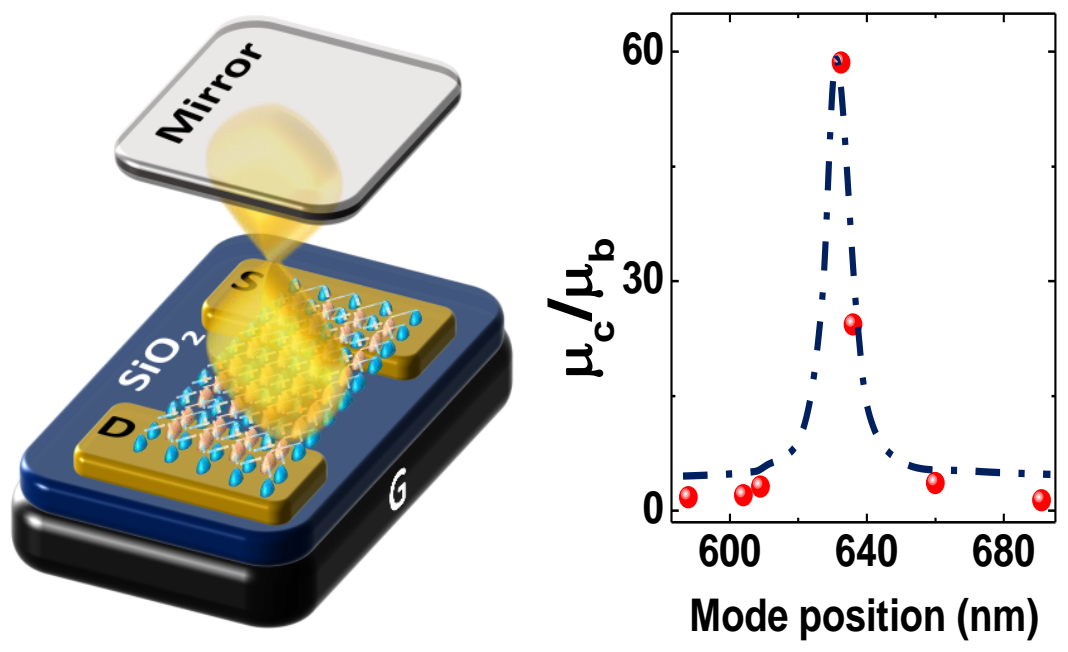

TOC 
2D materials based on transition metal dichalcogenides (TMDCs) are emerging class of materials due to its remarkable optoelectronic properties. ${ }^{1,2}$ For example, atomically thin $\mathrm{WS}_{2}$ has very large absorption cross-section, narrow linewidth as well as high electron mobility. $\frac{3}{-}$ These direct band gap materials are nowadays used in many device applications that range from biosensing to optoelectronics. ${ }^{4}$ High crystallinity, delocalized electrons and exciton absorption in the visible region are the salient features that make these $2 \mathrm{D}$ materials suitable for optoelectronic devices.. Along with these properties, TMDs are also used in valleytronics that are polarization sensitive. ${ }^{6}$ Strong light-matter coupling was introduced to tailor the energy level that affect material properties from superconductivity to energy transfer., $, 8,9,10$ So far, 2D material strong coupling was utilized in polariton lasing, spin selectivity and photoluminescence enhancement. $\frac{11,12,13,14,15}{15} \mathrm{WS}_{2}$ monolayers can be effectively coupled to a Fabry-Perot (FP) cavity due to its high transition dipole moment ( $>50$ Debye) that resulted in the generation of polaritonic states with splitting energy of the order of $100 \mathrm{meV} .^{16}$ Multilayer coupling scheme was also adopted to improve the coupling strength in these materials. ${ }^{17}$ Other approaches include the usage of plasmonic arrays, distributed Bragg reflectors (DBR) that can also give decent coupling strength to study the nature of hybrid light-matter states. ${ }^{18,19}$ So far, all the strong coupling studies in $2 \mathrm{D}$ materials are based on optical characterization and understanding the energy delocalization. ${ }^{20}$ Here, we used the idea of lightmatter strong coupling to improve the charge transport through polaritonic states in 2D materials.

Charge transport through the polaritonic state was first demonstrated by Ebbesen and co-workers in 2015..$^{21}$ Perylenediimide based organic material was coupled to plasmonic arrays, and the corresponding I-V characteristics was measured using a 2-terminal device configuration. Resonance coupling of the active layer to the plasmonic mode resulted in an enhancement of conductivity by an order of magnitude. This may result in the delocalization of molecular orbitals under strong coupling conditions that boost charge transport through the polaritonic states-this concept can be called as polaritronics..$^{22}$ There are mainly three mechanisms proposed for this observed phenomenon, that include: (i) effective mass of the polaritonic state, (ii) molecular coherence and (iii) work function modification under strong coupling. $.1, \underline{23}, \underline{24}, \underline{25}, \underline{26}$ Both experimental and theoretical studies suggest that either one or all of the above mechanisms may operate together to boost the transport in the medium. $\stackrel{21}{ }$ Organic electronics is based on hopping mechanism and hence molecular packing has a huge role in controlling their electrical properties. $\underline{27}, \underline{28}$ Previous observation also points to the fact that crystal packing is a necessary criterion to achieve better electron transport. ${ }^{29}$ In the current study, we used highly ordered $\mathrm{WS}_{2}$ multilayers with WannierMott excitons having narrow absorption bandwidth. These features, along with a high absorption cross-section, help us to study charge transport behaviour under strong coupling condition. Here, we used an unconventional configuration of Fabry-Perot (FP) cavity that works in a field-effect transistor (FET) configuration (Figure 1 and 2). Few layer $\mathrm{WS}_{2}$ is effectively coupled to the $\lambda$ cavity mode; both optical and electrical output is measured to understand the nature of transport in the strongly coupled system. 
$\mathrm{WS}_{2}$ monolayer is unique in its optical behaviour that shows the highest absorption cross-section as well as the high quantum yield of fluorescence. $\frac{30}{}$ Both monolayer and few-layer $\mathrm{WS}_{2}$ shows sharp exciton bands in the visible region (Figure 1). Monolayers are direct bandgap materials, whereas multilayers show indirect bandgap. Due to spin-orbit coupling, the valance band split into two states in K-space, resulting in two optical transitions, shown in Figure 1a, c. A-exciton show powerful absorption features compared to the B-exciton and is utilized for strong coupling experiments. ${ }^{16} \mathrm{~A}$ few-layer $\mathrm{WS}_{2}$ flake is coupled to $\lambda$ mode of the cavity generating hybrid lightmatter states with Rabi splitting energy of $95 \mathrm{meV}$. Here, the cavity is fabricated using a silicon wafer (with $90 \mathrm{~nm} \mathrm{SiO} 2$ coating) and a thin metal mirror (Ag; $30 \mathrm{~nm}$ ) having poly-methyl methacrylate (PMMA) as a tunable spacer (Figure 1b). Introduction of a few-layer ( 6 layer) freshly exfoliated $\mathrm{WS}_{2}$ at the anti-node position resulted in the splitting of the energy level, which generate two polaritonic states ( $\mathrm{P}+$ and $\mathrm{P}-$ ). Dispersion experiment gives a clear anti-crossing with the splitting energy of $95 \mathrm{meV}$ as the light-line crosses the A-exciton transition (Figure 1d). In order to understand the nature of the interaction, we have calculated the electromagnetic field distribution using the transfer matrix method (TMM). TMM studies suggest localized modes with a slight penetration of field into the silicon (Figure S1 and S2; Section 1; SI). The cavity modes are dissipative with a Q-factor of $\sim 10$, whereas, the splitting energy is approximately the same as compared to the typical FP cavity configuration reported in the literature. $\underline{17}, \underline{31}$

Further, we explored the effect of thickness of the active layer on the coupling strength. Different cavities are fabricated using monolayer to few-layer $\mathrm{WS}_{2}$. It is reported that the A-exciton peak position moves from $613 \mathrm{~nm}$ to $629 \mathrm{~nm}^{\frac{17}{17}}$ for monolayer to few-layer system due to Coulomb screening effect in a multilayer structure (Figure 2a). Tuning cavity mode to A-exciton peak position and Rabi splitting energy was measured by placing the active layer precisely at the antinode of the cavity (Figure 2b). The splitting energy varies non-linearly due to change in the effective transition dipole moment of a few-layer $\mathrm{WS}_{2}$ (Figure 2c). In-plane transition dipole moment gradually decreases and saturate for a multilayer, whereas, the in-plane field distribution still enhances the strong coupling contribution in the system. These results are exactly similar to the previous studies reported by Wang et.al, in a nanoparticle array configuration. $\frac{17}{}$

The electrical properties of the coupled system are measured in 3-terminal, bottom gate FET configuration as shown in Figure 3a. An active layer of $\mathrm{WS}_{2}$ is placed in between the $\mathrm{SiO}_{2}$ and PMMA layer to achieve ON resonance condition. $\mathrm{n}^{++}$dopped $\mathrm{Si}$ is used as the substrate for the electrical measurements (see experimental methods). $\mathrm{SiO}_{2}$ layer was thermally evaporated to achieve a specific thickness of $90 \mathrm{~nm}$. Ti/Au electrodes $(10 / 30 \mathrm{~nm})$ were deposited on $\mathrm{SiO}_{2}$ to complete the 3-terminal configuration. Few-layer $\mathrm{WS}_{2}$ was exfoliated using scotch tape method and transferred on Au electrode via PDMS substrate. In the next step, Ag mirror (30 nm) was deposited on a PDMS slab followed by spin-coating of PMMA. The thickness of the PMMA is controlled by varying the rotation speed. Now, PMMA coated Ag mirror is bonded on top of the $\mathrm{WS}_{2}$ layer by physical adsorption. This completes the FP cavity in FET configuration; the active layer is now positioned at one of the anti-nodes of the $\lambda$ cavity. Source and drain separation is 
fixed at a length of $\mathrm{L}=5 \mu \mathrm{m}$, and width of $\mathrm{WS}_{2}$ at $\sim 5.5 \mu \mathrm{m}$ (changes depending on the dimension of the $\mathrm{WS}_{2}$ flake transferred for each device; these parameters are taken for the mobility calculations). AFM section analysis suggested the thickness of the active layer as $6.2 \mathrm{~nm}$, suggesting a 6-layer $\mathrm{WS}_{2}$ device (Figure $3 \mathrm{~b}$ ).

The above device was then taken into an inert gas glove box and annealed at $200^{\circ} \mathrm{C}$ to remove any organic impurities. I-V characteristics are measured in an inert atmosphere, as shown in Figure $3 \mathrm{c}$ (black points). ON/OFF resonance data were acquired by introducing the mirror with varying PMMA thickness on the same sample in repeated measurements. Figure $3 \mathrm{c}$ is a representative $\mathrm{I}_{\mathrm{d}^{-}}$ $\mathrm{V}_{\mathrm{d}}$ curve (gate voltage; $\mathrm{V}_{\mathrm{g}}=0 \mathrm{~V}$ ) for bare, $\mathrm{OFF}$ and $\mathrm{ON}$ resonance systems using the same $\mathrm{WS}_{2}$ flake as an active layer. Please note that annealing of bare flake is necessary before each measurement, and annealing after the bonding is not recommended. This minimizes the error in the electrical measurements and gets good consistency of the data. In all the structures, we observed a positive gating effect with a non-linear $\mathrm{I}_{\mathrm{d}}-\mathrm{V}_{\mathrm{d}}$ characteristics at different $\mathrm{V}_{\mathrm{g}}$ (Figure $3 \mathrm{~d}$ ). It shows Schottky contact between metal/ $\mathrm{WS}_{2}$ interface in the device. Mobility of the bare flake is extracted from the transfer characteristic of the device and is approximately $5 \times 10^{-2} \mathrm{~cm}^{2} \mathrm{~V}^{-1} \mathrm{~s}^{-1}$ (Table S1; section 2; SI). The mobility of the bare device is low due to very high Schottky contact and also due to the presence of a dielectric layer $\left(\mathrm{SiO}_{2}\right)$ creating large number of trap states. Very interestingly, introducing a mirror on top of the device and slowly moving the system from OFF to $\mathrm{ON}$ resonance increases the mobility to $\sim 3 \mathrm{~cm}^{2} \mathrm{~V}^{-1} \mathrm{~s}^{-1}$ (Figure $3 \mathrm{e}$ ). $\mathrm{I}_{\text {on }} / \mathrm{I}_{\text {off }}$ ratio of the ON resonance cavity is increased by 2 orders of magnitude, suggesting a better performance of the device under strong coupling condition.

In the next step, mobility was measured while tuning the cavity mode position. A clear enhancement in mobility is observed while the active layer is efficiently coupled to the $\lambda$ cavity mode. In order to understand the strong coupling effect, the ratio of mobility is plotted against the cavity mode position (Figure 4a). Mobility of the active layer is boosted approximately 50 times at $\mathrm{ON}$ resonance condition, and the change in mobility follows the line-shape of the A-exciton band of $\mathrm{WS}_{2}$. The effective mass of the polaritonic state $(\mathrm{P}-)$ is calculated from the optical detuning experiments. The effective mass change is $\sim 10^{-5}$ at $\mathrm{ON}$ resonance condition compared to the bare structure (Figure S4a). In order to understand this large variation in mobility, we extracted the change in Schottky barrier height (SBH) using the thermionic electron transport relation,

$$
\Phi_{c}-\Phi_{b}=\frac{k T}{e} \ln \left(\frac{I_{b}}{I_{c}} \frac{m_{c}^{*}}{m_{b}^{*}}\right)
$$

where, $\Phi_{c}$ and $\Phi_{b}$ are SBH for cavity and bare structures. $I_{c}$ and $I_{b}$ are drain current for cavity and bare device at same bias voltage. $m_{c}{ }^{*}$ and $m_{b}{ }^{*}$ are the effective mass of lower polaritonic state $(\mathrm{P}-$ ) and bare electron in few-layer $\mathrm{WS}_{2}$, respectively (section 3; SI). SBH decreased by $45 \mathrm{meV}$ under ON resonance condition (Figure 4b). Change in SBH against the cavity mode position follows the line shape of $\mathrm{WS}_{2}$, indicating a clear signature of strong coupling effect in the charge transport measurements. 
Here, the effective mass calculated from the optical dispersion of the coupled system is comparable to the current density flow in the device. Light-matter strong coupling can boost the delocalization across the Wannier-Mott excitons in $\mathrm{WS}_{2}$, generating an improved flow of electrons through the polaritonic states. Few of the experimental and theoretical measurements of strongly coupled systems suggest lower electron scattering, lattice coherence, and collective nature of the states. $2,32,33, \underline{34}$ Another attempt was to explain the effect of strong coupling to bypass the disordered lattice that improves the charge transport. In general, all the experimental findings are suggesting a better coherence in the coupled system. In the current work, we used the effective mass of lower polaritonic state (optical output) as a handle to incorporate coherence parameter and calculated the change in SBH of the coupled system using measured current density (electrical output). Our results propose a dispersion behavior of SBH in strongly coupled system (Figure 4c). This observation is a clear indication of the hybrid nature of the polaritonic states.

In conclusion, we studied both optical and electrical properties of 2D materials under strong coupling condition. FP cavity in FET configuration is used here to understand the charge transport properties of the coupled system. Charge transport mobility is enhanced by $\sim 50$ times under ON resonance condition. Similarly, strong coupling can improve the $\mathrm{I}_{\mathrm{on}} / \mathrm{I}_{\text {off }}$ ratio of the FET device without chemical and mechanical modification of the active layer. Lattice coherence and work function variation are the key factors that control charge transport in strongly coupled system. Our results emphasize the potential of light-matter strong coupling in material science.

\section{Experimental Methods:}

Device preparation: $\mathrm{WS}_{2}$ flakes were mechanically exfoliated on PDMS substrate by scotch tape method from bulk crystal (purchased from HQ-Graphene, The Netherlands). Exfoliated $\mathrm{WS}_{2}$ flake of desired thickness on PDMS was selected initially by optical contrast using white light/halogen lamp illumination and later by AFM. $\mathrm{n}++$ doped silicon wafer with $90 \mathrm{~nm} \mathrm{SiO}_{2}$ and 10/30 nm $\mathrm{Ti} / \mathrm{Au}$ electrodes pattern were purchased from Fraunhofer-IPMS, Germany. Next, desired flakes were transferred on the Ti/Au electrode $(\mathrm{L}=5 \mu \mathrm{m})$ by surface adhesion using a home-built transfer stage (dry-transfer). Samples were kept in an inert gas glove box and annealed at $200^{\circ} \mathrm{C}$ for 2 hours prior to electrical measurements. FP cavity in the FET configuration was prepared by sputtering (40 mA; BT300, Hind High-Vacuum) Ag mirror on a thick PDMS substrate. Poly-methyl methacrylate (PMMA;120 kDa; Sigma-Aldrich) in anisole was spin-coated (LABSPIN 6, SussMicrotech) on Ag mirror to achieve different thicknesses. PMMA coated Ag mirror was carefully placed on the bare FET device having active layer $\mathrm{WS}_{2}$.

Electrical/Optical characterization: All electrical measurements were done in the glove box (1 ppm $\mathrm{H}_{2} \mathrm{O}$ and 1 ppm $\mathrm{O}_{2}$ ) using $\mathrm{MB}$ 150, Cascade Microtech probe station. Nikon inverted microscope (Eclipse Ti2) was used for optical measurements. Optical measurements are carried out using white light LED (transmission) and halogen lamps (reflection). Transmitted/reflected light was collected through the objective $(20 \mathrm{X}, \mathrm{NA}=0.45)$ to a spectrometer (SpectraPro HRS300 , Princeton Instruments) coupled to liquid nitrogen cooled at $-120^{\circ} \mathrm{C}$ charge-coupled device 
(CCD) camera (Pylon 100BX) for spectral acquisition. All the measurements were done at room temperature.

\section{AUTHOR INFORMATION}

Corresponding Author

* jgeorge@iisermohali.ac.in

\section{Funding Sources}

DST-SERB, Core Research Grant (EMR/2017/003455), and MHRD-Scheme for Transformational and Advanced Research in Sciences (MoE-STARS/STARS-1/ 175).

\section{ACKNOWLEDGMENT}

P.B. and K. K. thank IISER Mohali for the fellowship.

\section{REFERENCES}

(1) Manzeli, S.; Ovchinnikov, D.; Pasquier, D.; Yazyev, O. V.; Kis, A. Nature Reviews Materials 2017, 2, 17033.

(2) Xia, F.; Wang, H.; Xiao, D.; Dubey, M.; Ramasubramaniam, A. Nat Photonics 2014, 8, 899.

(3) Nazir, G.; Khan, M. F.; Iermolenko, V. M.; Eom, J. RSC Advances 2016, 6, 60787.

(4) Mueller, T.; Malic, E. npj 2D Materials and Applications 2018, 2, 29.

(5) Jo, S.; Ubrig, N.; Berger, H.; Kuzmenko, A. B.; Morpurgo, A. F. Nano Lett 2014, 14, 2019.

(6) Mai, C.; Barrette, A.; Yu, Y.; Semenov, Y. G.; Kim, K. W.; Cao, L.; Gundogdu, K. Nano Lett 2014, 14, 202.

(7) Thomas, A.; Devaux, E.; Nagarajan, K.; Chervy, T.; Seidel, M.; Hagenmüller, D.; Schütz, S.; Schachenmayer, J.; Genet, C.; Pupillo, G. arXiv preprint arXiv:1911.01459 2019.

(8) Schlawin, F.; Cavalleri, A.; Jaksch, D. Physical Review Letters 2019, 122, 133602.

(9) Zhong, X.; Chervy, T.; Wang, S.; George, J.; Thomas, A.; Hutchison, J. A.; Devaux, E.; Genet, C.; Ebbesen, T. W. Angewandte Chemie International Edition 2016, 55, 6202.

(10) Coles, D. M.; Somaschi, N.; Michetti, P.; Clark, C.; Lagoudakis, P. G.; Savvidis, P. G.; Lidzey, D. G. Nature Materials 2014, 13, 712.

(11) Cheng, C.-Y.; Dhanker, R.; Gray, C. L.; Mukhopadhyay, S.; Kennehan, E. R.; Asbury, J. B.; Sokolov, A.; Giebink, N. C. Physical Review Letters 2018, 120, 017402.

(12) Gu, J.; Chakraborty, B.; Khatoniar, M.; Menon, V. M. Nat Nanotechnol 2019, 14, 1024. 
(13) Javerzac-Galy, C.; Kumar, A.; Schilling, R. D.; Piro, N.; Khorasani, S.; Barbone, M.; Goykhman, I.; Khurgin, J. B.; Ferrari, A. C.; Kippenberg, T. J. Nano Lett 2018, 18, 3138.

(14) Latini, S.; Ronca, E.; De Giovannini, U.; Hübener, H.; Rubio, A. Nano Lett 2019, 19, 3473.

(15) Chervy, T.; Azzini, S.; Lorchat, E.; Wang, S.; Gorodetski, Y.; Hutchison, J. A.; Berciaud, S.; Ebbesen, T. W.; Genet, C. ACS Photonics 2018, 5, 1281.

(16) Wang, S.; Li, S.; Chervy, T.; Shalabney, A.; Azzini, S.; Orgiu, E.; Hutchison, J. A.; Genet, C.; Samorì, P.; Ebbesen, T. W. Nano Lett 2016, 16, 4368.

(17) Wang, S.; Le-Van, Q.; Vaianella, F.; Maes, B.; Eizagirre Barker, S.; Godiksen, R. H.; Curto, A. G.; Gomez Rivas, J. ACS Photonics 2019, 6, 286.

(18) Liu, X.; Galfsky, T.; Sun, Z.; Xia, F.; Lin, E.-c.; Lee, Y.-H.; Kéna-Cohen, S.; Menon, V. M. Nat Photonics 2015, 9, 30.

(19) Schneider, C.; Glazov, M. M.; Korn, T.; Höfling, S.; Urbaszek, B. Nature Communications 2018, 9, 2695.

(20) Low, T.; Chaves, A.; Caldwell, J. D.; Kumar, A.; Fang, N. X.; Avouris, P.; Heinz, T. F.; Guinea, F.; Martin-Moreno, L.; Koppens, F. Nature Materials 2017, 16, 182.

(21) Orgiu, E.; George, J.; Hutchison, J. A.; Devaux, E.; Dayen, J. F.; Doudin, B.; Stellacci, F.; Genet, C.; Schachenmayer, J.; Genes, C.; Pupillo, G.; Samorì, P.; Ebbesen, T. W. Nature Materials 2015, 14, 1123.

(22) Deveaud-Plédran, B. Nature 2008, 453, 297.

(23) Nagarajan, K.; George, J.; Thomas, A.; Devaux, E.; Chervy, T.; Azzini, S.; Joseph, K.; Jouaiti, A.; Hosseini, M. W.; Kumar, A.; Genet, C.; Bartolo, N.; Ciuti, C.; Ebbesen, T. W. Acs Nano 2020, $14,10219$.

(24) Hagenmüller, D.; Schachenmayer, J.; Schütz, S.; Genes, C.; Pupillo, G. Physical Review Letters 2017, 119, 223601.

(25) Feist, J.; Garcia-Vidal, F. J. Physical review letters 2015, 114, 196402.

(26) Hutchison, J. A.; Liscio, A.; Schwartz, T.; Canaguier-Durand, A.; Genet, C.; Palermo, V.; Samori, P.; Ebbesen, T. W. Adv Mater 2013, 25, 2481.

(27) Akselrod, G. M.; Deotare, P. B.; Thompson, N. J.; Lee, J.; Tisdale, W. A.; Baldo, M. A.; Menon, V. M.; Bulović, V. Nature Communications 2014, 5, 3646.

(28) Yan, H.; Chen, Z.; Zheng, Y.; Newman, C.; Quinn, J. R.; Dötz, F.; Kastler, M.; Facchetti, A. Nature 2009, 457, 679. 
(29)Vehoff, T.; Baumeier, B.; Troisi, A.; Andrienko, D. Journal of the American Chemical Society 2010, 132, 11702 .

(30) Gutiérrez, H. R.; Perea-López, N.; Elías, A. L.; Berkdemir, A.; Wang, B.; Lv, R.; LópezUrías, F.; Crespi, V. H.; Terrones, H.; Terrones, M. Nano Lett 2013, 13, 3447.

(31) Bhatt, P.; Dutta, J.; George, J. physica status solidi (RRL) - Rapid Research Letters 2021, 15, 2000580.

(32) Rozenman, G. G.; Akulov, K.; Golombek, A.; Schwartz, T. ACS Photonics 2018, 5, 105.

(33) Naudet-Baulieu, C.; Bartolo, N.; Orso, G.; Ciuti, C. New J Phys 2019, 21, 093061.

(34) Ciuti, C.; Bastard, G.; Carusotto, I. Phys Rev B 2005, 72, 115303. 


\section{Figure 1:}

(a) WS Excitons

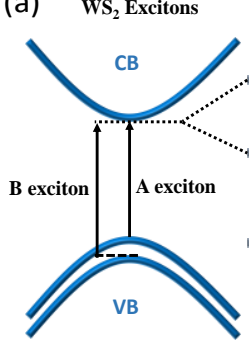

(c)

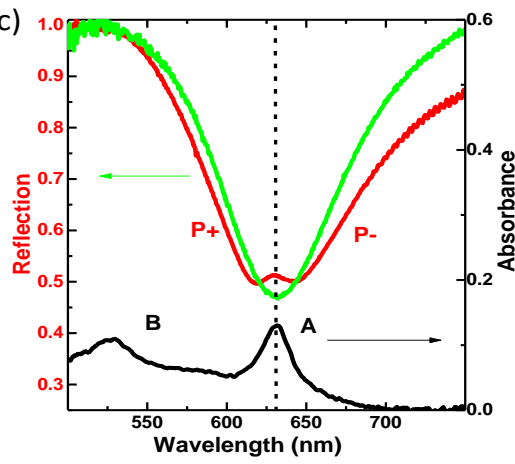
Light-matter

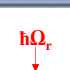

Cavity mode

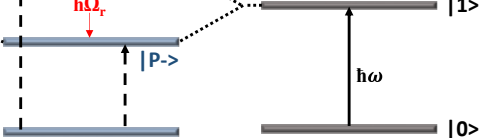

$|0\rangle$

(b)

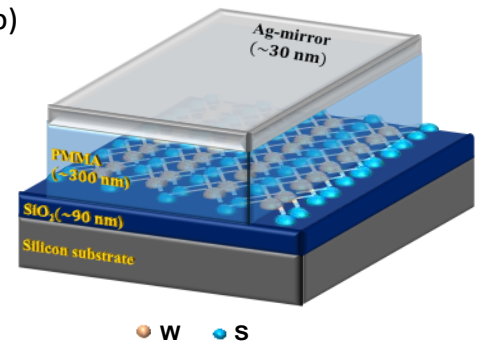

(d)

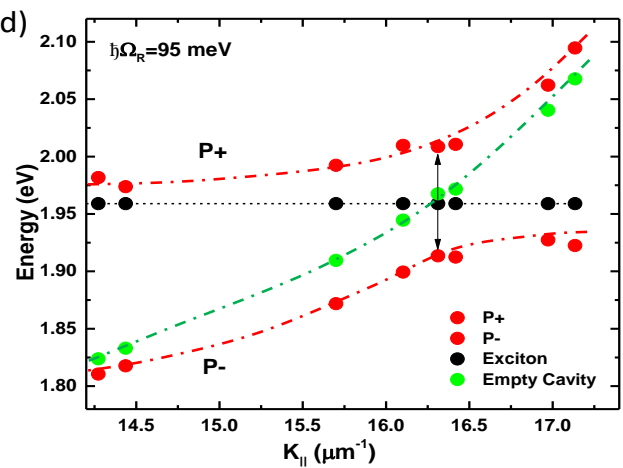

Figure 1. Optical spectra of FP cavity on Silicon wafer: (a) Schematic representation of formation of light-matter hybrid states between optical cavity mode and A-exciton of few-layer $\mathrm{WS}_{2}$. (b) Schematic showing FP cavity formed by one-mirror with embedded $\mathrm{WS}_{2}$ (here, $\mathrm{SiO}_{2}$ and $\mathrm{PMMA}$ act as spacers). (c) Absorbance spectrum of few-layer $\mathrm{WS}_{2}$ (6 layers; black solid line) and the corresponding polaritonic states $\mathrm{P}+$ and $\mathrm{P}$ - (red solid line) as a result of strong coupling between Aexciton of $\mathrm{WS}_{2}$ and $\lambda$ cavity mode (green solid line). (d) Experimental dispersion curve for corresponding strong coupled system shows $\mathrm{P}+$ and $\mathrm{P}$ - dispersion (red circles), dispersive empty cavity mode (green circles) and the position of A-exciton band (black circles) [dotted lines are guide to eye]. 


\section{Figure 2:}
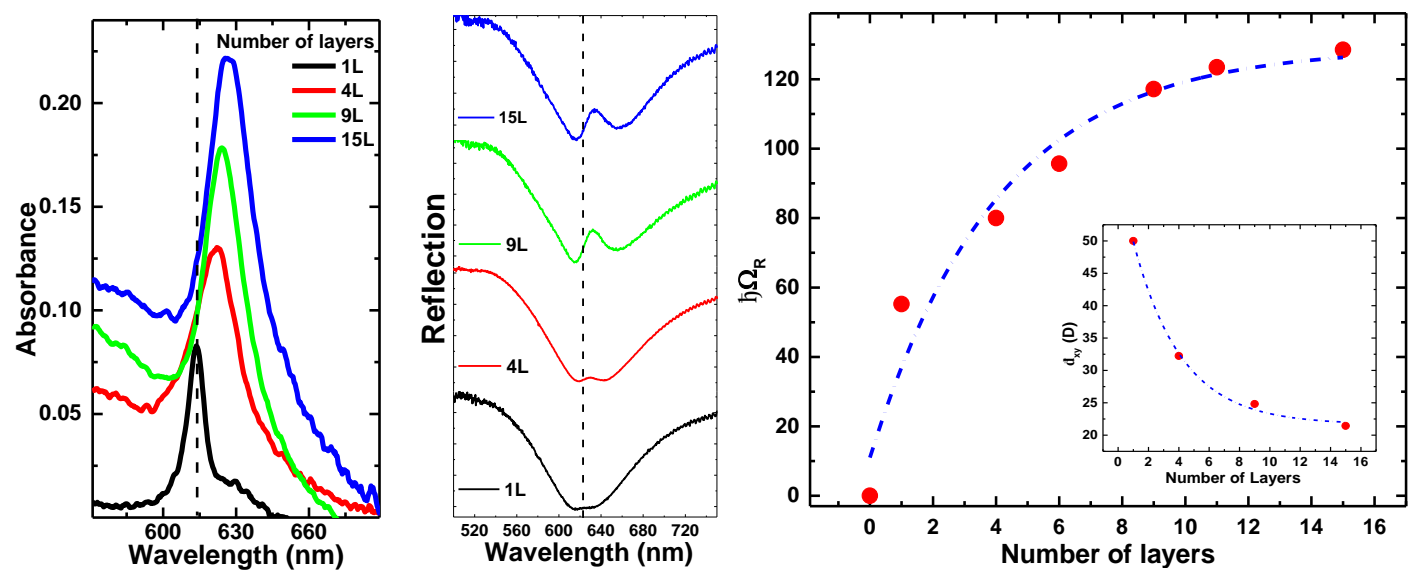

Figure 2. Rabi splitting versus number of WS layers: (a) Absorbance of $\mathrm{WS}_{2}$ for different layer thickness (1L, 4L, 9L and 15L), (b) the corresponding optical spectra of coupled system for one-mirror FP cavity on silicon. (c) Rabi splitting variation with $\mathrm{WS}_{2}$ layer thickness, when placed at antinode of the $\lambda$ cavity mode. Inset shows the in-plane dipole moment of A-exciton of $\mathrm{WS}_{2}$ versus number of layers. 
Figure 3:

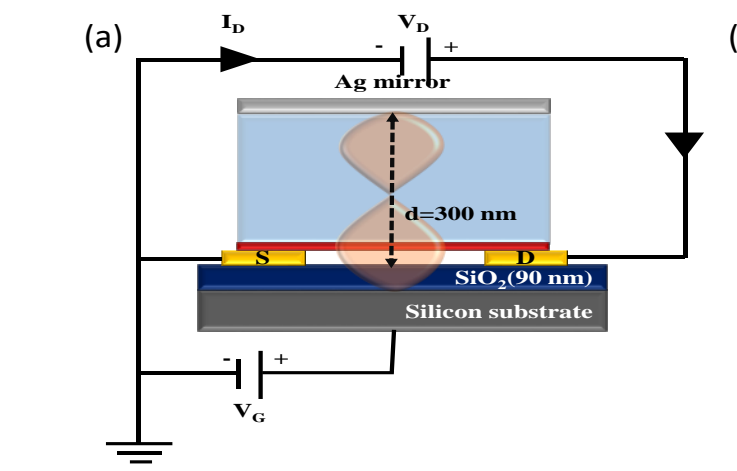

(b)

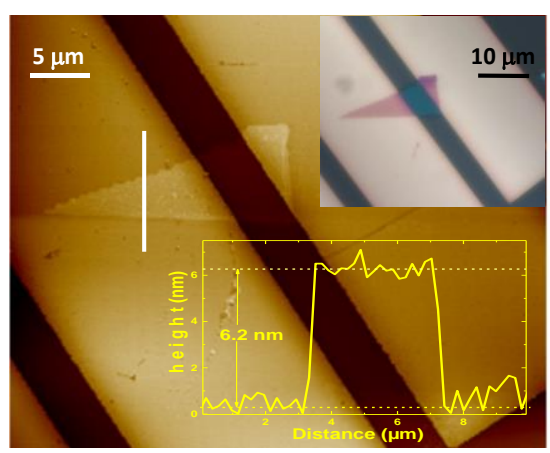

$54.4 \mathrm{~nm}$

(c)

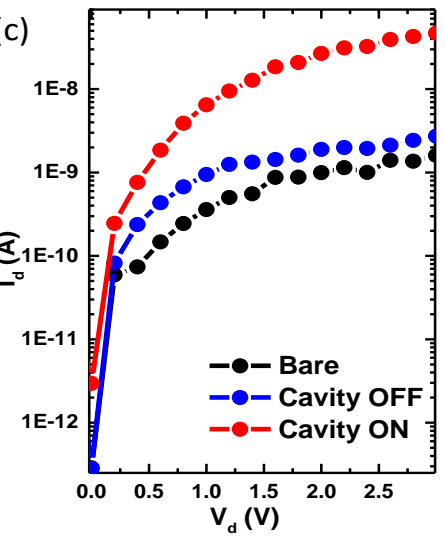

(d)

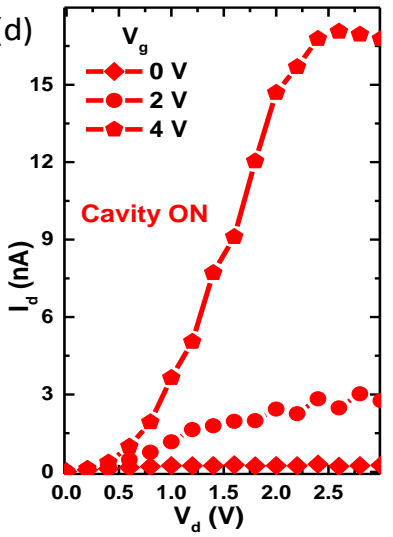

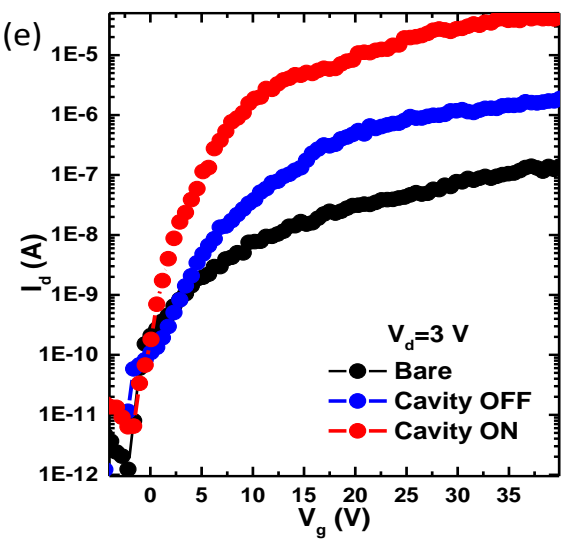

Figure 3. Electrical characterization of few-layer WS $\mathbf{W S}_{2}$ in FET configuration: (a) Pictorial representation of a 3 -terminal FET configuration of few-layer $\mathrm{WS}_{2}$ coupled to $\lambda$ cavity mode on silicon substrate with $90 \mathrm{~nm} \mathrm{SiO}_{2}$ and PMMA as spacer. (b) AFM image of the device having few-layer $\mathrm{WS}_{2}$ transferred on $\mathrm{Ti} / \mathrm{Au}$ electrode with $\mathrm{L}=5 \mu \mathrm{m}$ and $\mathrm{W}=5.5 \mu \mathrm{m}$. Inset shows the optical image for same device and height trace of $\sim 6.2 \mathrm{~nm}$ for few-layer $\mathrm{WS}_{2}$. (c) $\mathrm{I}_{d}-\mathrm{V}_{\mathrm{d}}$ for the bare (black circles), OFF resonance (blue circles) and $\mathrm{ON}$ resonance (red circles) FET device at $\mathrm{Vg}=0 \mathrm{~V}$. (d) $\mathrm{I}_{\mathrm{d}}-\mathrm{V}_{\mathrm{d}}$ characteristics of $\mathrm{ON}$ resonance $\mathrm{WS}_{2}$ at different gate voltages. (e) Transfer properties of $\mathrm{WS}_{2}$ for bare (black circles), OFF resonance (blue circles) and $\mathrm{ON}$ resonance (red circles) coupled to $\lambda$ cavity mode. 


\section{Figure 4:}
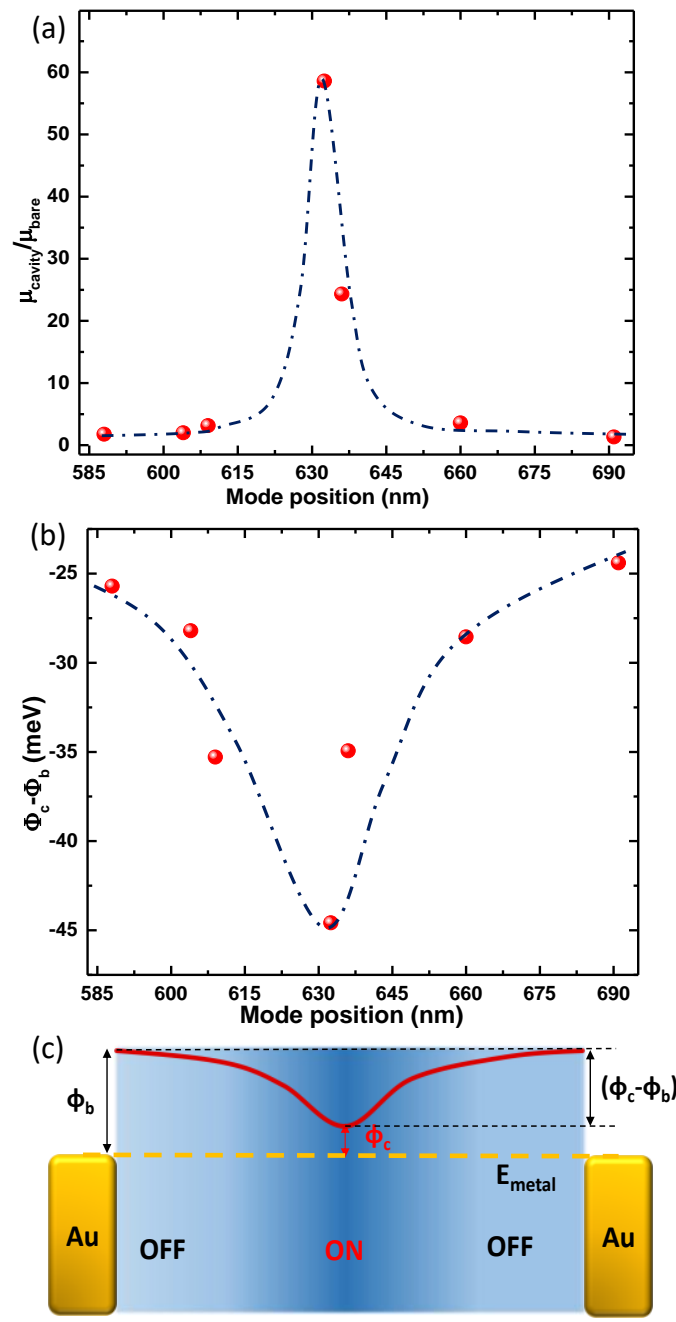

Figure 4. Cavity tuning and mobility enhancement: (a) Mobility enhancement for $\lambda$ cavity tuning, ON resonance situation shows maximum enhancement of mobility. (b) Change in $\mathrm{SBH}$ from bare structure calculated $\left(\mathrm{V}_{\mathrm{g}}=0 \mathrm{~V} ; \mathrm{V}_{\mathrm{d}}=3 \mathrm{~V}\right)$ for OFF to $\mathrm{ON}$ resonance cavity. (c) Schematic showing cavity effect on $\mathrm{SBH}$ of few-layer $\mathrm{WS}_{2}$ under strong coupling. This illustrates the changes in $\mathrm{SBH}$ is maximum at $\mathrm{ON}$ resonance condition. 


\title{
Supporting Information
}

\section{Enhanced Charge Transport in 2D materials through Polaritonic States}

\author{
Pooja Bhatt, Kuljeet Kaur and Jino George* \\ Indian Institute of Science Education and Research (IISER) Mohali, Punjab-140306, India. \\ *'jgeorge@iisermohali.ac.in
}

\section{Contents:}

SI. No. $\quad$ Name of the section

1 Transfer Matrix Method (TMM) Simulation

2 Electrical characterization

3 Effective mass and SBH calculations

$4 \quad$ Supporting References 


\section{Transfer Matrix Method (TMM) Simulation:}

Transfer matrix method (TMM) is a semiclassical tool to study interference from multilayer structures. It calculates transmission and reflection coefficients at the interface of the medium having different refractive indices, using Fresnel equations and plane wave solutions of Maxwell equations in a given medium. To understand cavity systems $\mathrm{Silicon} / \mathrm{SiO}_{2} / \mathrm{WS}_{2} / \mathrm{PMMA} / \mathrm{Ag}$ stack

(a)

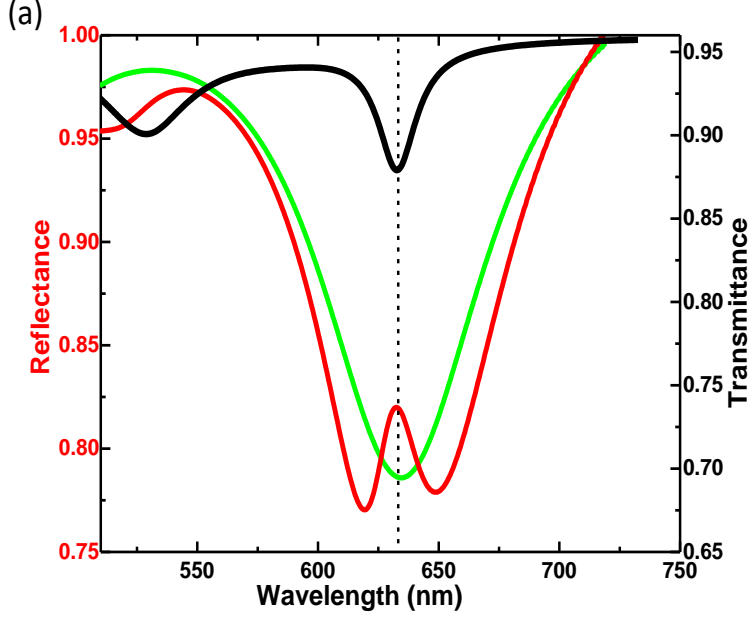

(b)

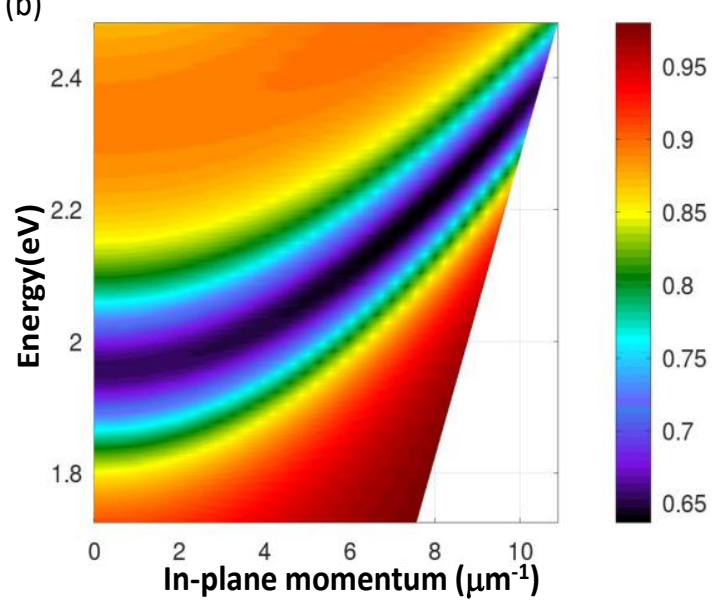

Figure S1. (a) TMM calculations: transmittance of few-layer $\mathrm{WS}_{2}$ fitted to experimental transmission (black solid line), empty cavity spectra (green solid line) and few-layer $\mathrm{WS}_{2}$ coupled to one mirror cavity. (b) Corresponding empty cavity TMM calculated dispersion.

was studied in TMM simulations. Ag mirror thickness of $\approx 30 \mathrm{~nm}$ (refractive index of silver is taken from Johnson and Christy, 1972) was used for all studies. ${ }^{1}$
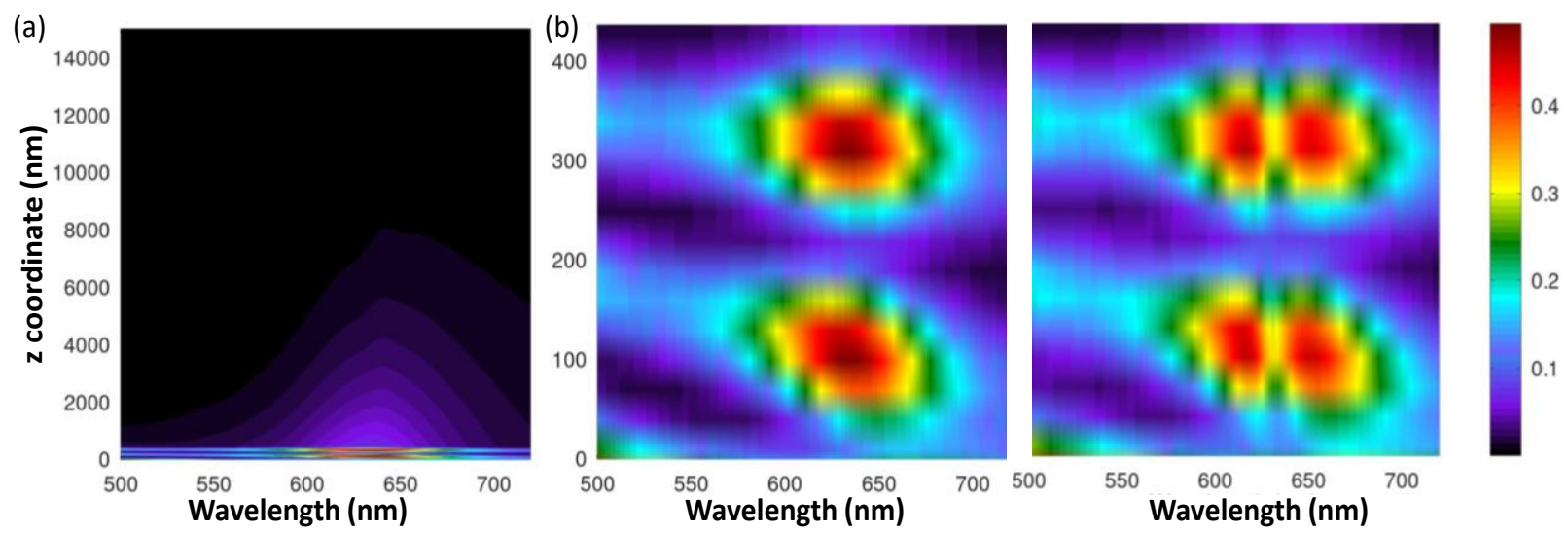

Figure S2. (a) Electric field distribution of empty cavity mode on Ag (one mirror)/silicon-based FP cavity shows penetration of electric field into the silicon substrate. (b) Electric field distribution (zoomed part between $\mathrm{Ag}$ mirror and silicon) for empty $\lambda$ mode (left), and after coupling with a few-layer $\mathrm{WS}_{2}$ (right). 
Figure $\mathrm{S} 1(\mathrm{a})$ is the TMM fitted transmission spectrum for few-layer $\mathrm{WS}_{2}$ and empty $\lambda$ cavity mode resonantly coupled to few-layer $\mathrm{WS}_{2} \mathrm{~A}$-exciton band. Placing few-layer $\mathrm{WS}_{2}$ at one of the antinodes of the $\lambda$ cavity, giving the splitting of $\sim 95 \mathrm{meV}$ from TMM simulations which are in agreement with experiments. Corresponding empty TMM calculated dispersion is shown in figure $\mathrm{S} 1$ (b). For simulations, $\mathrm{SiO}_{2}$ thickness was $\sim 90 \mathrm{~nm}$ and PMMA $\sim 300 \mathrm{~nm}$ at the ON resonance condition. It can be seen from the field distribution profile (figure S2(a)), there is small penetration of electromagnetic field toward the silicon substrate. $\mathrm{WS}_{2}$ is efficiently coupled to $\lambda$ cavity mode in the given condition (figure S2(b)).

\section{Electrical characterization:}

$I_{d}-V_{d}$ characteristics of bare and OFF resonance cavity device are shown figure S3(a) and S3(b) respectively. Electron transport at room temperature was calculated from the linear regime field effect mobility formula:
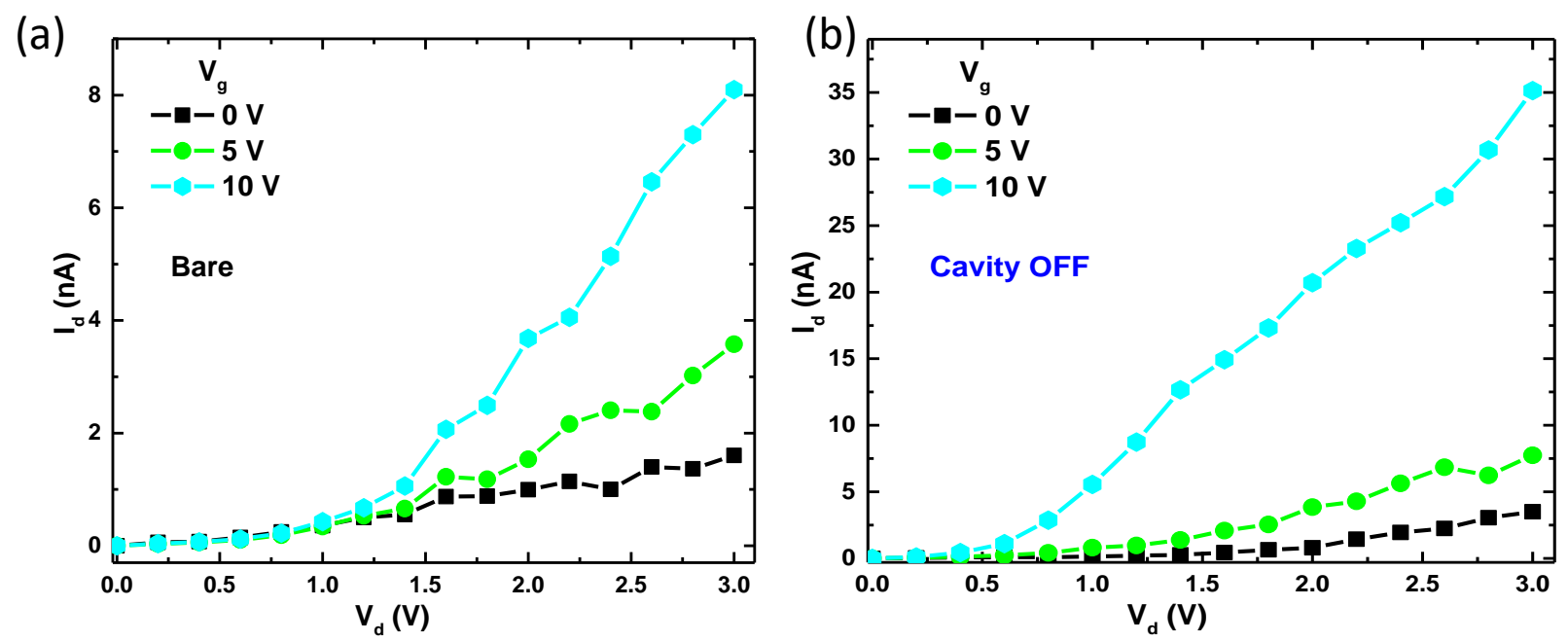

Figure S3. $\mathrm{I}_{\mathrm{d}}-\mathrm{V}_{\mathrm{d}}$ characteristics for (a) bare and (b) OFF resonance cavity device shown in main text, figure(3).

$$
\mu=\frac{L}{W C_{o x} V_{d}} \frac{d I_{d}}{d V_{g}}
$$

Where, $W$ and $L$ are channel width and length respectively, $C_{o x}$ is oxide layer capacitance can be calculated from $C_{o x}=\frac{\varepsilon_{0} \varepsilon_{r}}{d_{i}}$. Here $\varepsilon_{r}=3.9$ for $\mathrm{SiO}_{2}$ and $d_{i}=90 \mathrm{~nm}$. 
Table S1. Calculated mobility for OFF resonance bare and cavity FET device.

\begin{tabular}{|c|c|c|c|}
\hline $\begin{array}{c}\text { Cavity Mode position } \\
(\mathbf{n m})\end{array}$ & $\begin{array}{c}\boldsymbol{\mu}_{\text {bare }} \\
\left(\mathbf{c m}^{\mathbf{2}} \boldsymbol{V}^{-\mathbf{1}} \boldsymbol{s}^{\mathbf{1}}\right)\end{array}$ & $\begin{array}{c}\boldsymbol{\mu}_{\text {cavity }} \\
\left(\mathbf{c m}^{\mathbf{2}} \boldsymbol{V}^{\mathbf{- 1}} \boldsymbol{s}^{\mathbf{1}}\right)\end{array}$ & $\boldsymbol{\mu}_{\text {cavity }} / \boldsymbol{\mu}_{\text {bare }}$ \\
\hline 588 & $3.1 \times 10^{-2}$ & $5.6 \times 10^{-2}$ & 1.81 \\
\hline 604 & $5.5 \times 10^{-2}$ & $10.6 \times 10^{-2}$ & 1.92 \\
\hline 609 & $2.9 \times 10^{-2}$ & $9.5 \times 10^{-2}$ & 3.28 \\
\hline 633 & $5.2 \times 10^{-2}$ & $3.0 \times 10^{0}$ & 58.00 \\
\hline 636 & $3.2 \times 10^{-2}$ & $78.6 \times 10^{-2}$ & 24.56 \\
\hline 660 & $2.5 \times 10^{-2}$ & $8.9 \times 10^{-2}$ & 3.56 \\
\hline 691 & $10 \times 10^{-2}$ & $13.2 \times 10^{-2}$ & 1.32 \\
\hline
\end{tabular}

\section{Effective mass and SBH calculations:}

Effective mass for the lower polaritonic state (P-) can be calculated using Hopfield coefficients. ${ }^{2}$

$$
\frac{1}{m_{L P}}=\frac{\left|X\left(k_{\|}\right)\right|^{2}}{m_{e}}+\frac{\left|C\left(k_{\|}\right)\right|^{2}}{m_{c a v}}
$$

(a)

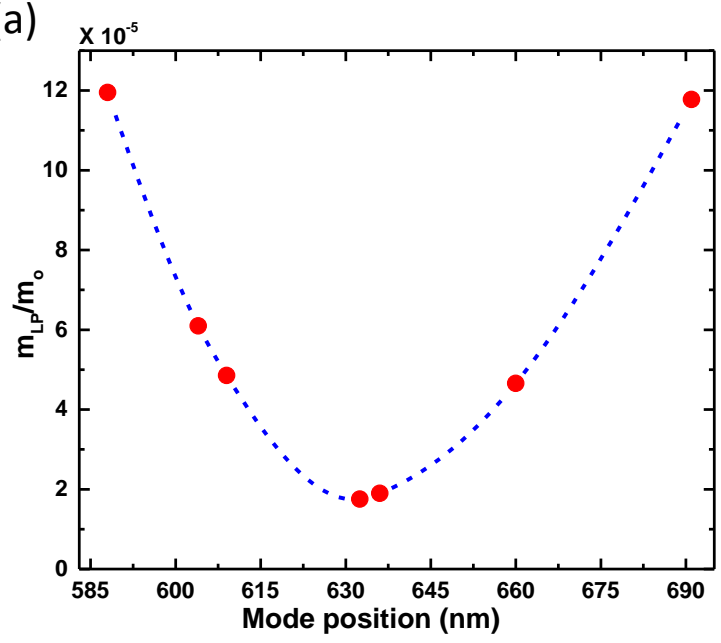

(b)

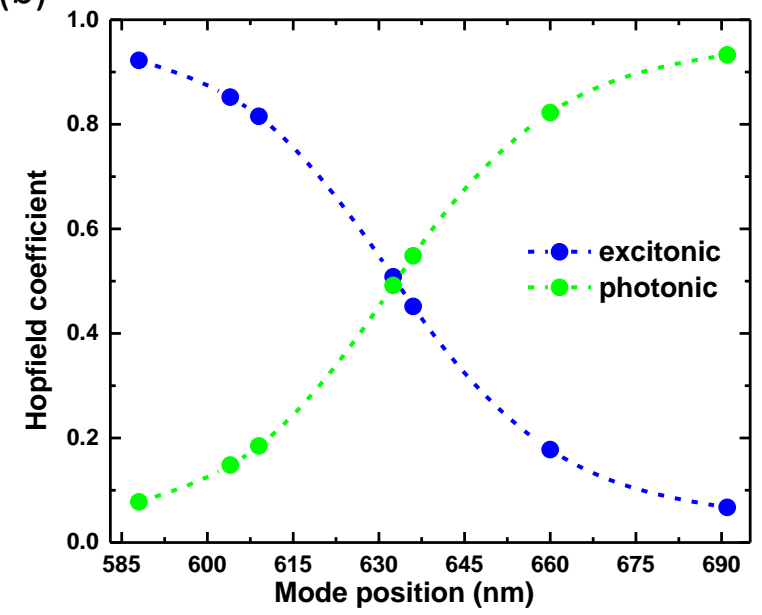

Figure S4. (a) Variation of effective mass and (b) Hopfield coefficients for lower polaritonic state $(\mathrm{P}-)$ as a function of cavity mode position (dotted lines are guide to eye).

Where, $m_{L P}$ is the effective masses of lower polaritonic states, respectively. $\mathrm{m}_{\mathrm{e}} \approx 1.096 \mathrm{~m}_{0}$ (electron reduced mass for few-layer $\left.\mathrm{WS}_{2}\right),{ }^{3}$ where $m_{0}$ is the free electron mass and $\mathrm{m}_{\text {cav }}$ is the cavity-photon effective mass which is given by; 


$$
m_{c a v}=\frac{E_{c a v}\left(k_{\|}\right)}{c^{2} / n_{c}^{2}}
$$

Hopfield coefficients and electron effective mass as function of cavity mode position calculated from optical measurements is shown in figure $\mathrm{S} 4$ (a) and $\mathrm{S} 4$ (b).

For calculation of SBH thermionic diode current equation was used, ${ }^{4}$

$$
I_{d}=A A^{*} e^{-e \Phi_{S B H} / k T}\left(e^{e V_{d} / k T}-1\right)
$$

Where, $I_{d}$ and $V_{d}$ are drain current and drain voltage respectively, $e$ is the electron charge, $k$ is Boltzmann constant, $T$ is absolute temperature, $\Phi_{S B H}$ is $\mathrm{SBH}$, and $A^{*}$ is Richardson constant. From above equation $\mathrm{SBH}$ can be extracted as follows,

$$
\Phi_{S B H}=\frac{k T}{e} \ln \left(\frac{A A^{*}\left(e^{e V_{d} / k T}-1\right)}{I_{d}}\right)
$$

Here, the Richardson constant depends on the effective mass of electrons $A^{*}=\frac{4 \pi e k^{2} m^{*}}{h^{3}} .{ }^{5}$ Hence difference in SBH for the cavity $\left(\Phi_{c}\right)$ and few-layer $\mathrm{WS}_{2}\left(\Phi_{b}\right)$ can be calculated easily using SBH equation at the constant gate and drain voltage as following,

$$
\Phi_{c}-\Phi_{b}=\frac{k T}{e} \ln \left(\frac{I_{b}}{I_{c}} \frac{m_{c}^{*}}{m_{b}^{*}}\right)
$$

Where, $I_{c}$ and $I_{b}$ are drain current for cavity and bare device at same bias voltage. $m_{c}{ }^{*}$ and $m_{b}{ }^{*}$ are the effective mass of lower polaritonic state (P-) and bare electron in few-layer $\mathrm{WS}_{2}$, respectively.

\section{Supporting References:}

1. Johnson, P. B.; Christy, R.-W. Physical review B 1972, 6, (12), 4370.

2. Bhatt, P.; Dutta, J.; George, J. physica status solidi (RRL) - Rapid Research Letters 2021, 15, (4), 2000580.

3. Liu, Y.; Hu, X.; Wang, T.; Liu, D. ACS Nano 2019, 13, (12), 14416-14425.

4. Kim, H.-C.; Kim, H.; Lee, J.-U.; Lee, H.-B.; Choi, D.-H.; Lee, J.-H.; Lee, W. H.; Jhang, S.

H.; Park, B. H.; Cheong, H.; Lee, S.-W.; Chung, H.-J. ACS Nano 2015, 9, (7), 6854-6860.

5. Khan, A. R.; Lu, T.; Ma, W.; Lu, Y.; Liu, Y. Advanced Electronic Materials 2020, 6, (4), 1901381. 